\title{
Superconducting Magnets for Toroidal Fields
}

\section{A multinational exploration of different design concepts}

\author{
P.N. Haubenreich, Oak Ridge \\ (Project Manager, Large Coil Task; now Secretary of ITER Council, IAEA, Vienna)
}

The Large Coil Task (LCT), a ten-year programme of international collaboration in developing superconducting magnets, has been sucessfully concluded. Analyses of the experimental data are complete and a rather comprehensive report has just been published [1]. LCT participants are acclaiming the programme's achievements as a large step towards practical fusion power.

By the mid 1970's, it was generally recognized that superconducting magnets are a practical necessity for sustained deuterium-tritium burning in tokamaks. Substantial development needs were foreseen because of both the great size and high fields and the tokamak's toroidal configuration which results in field and force distributions that pose difficult design problems. Stability and reliability also had to be demonstrated. Considering the limitations of single-coil tests, the Oak Ridge National Laboratory conceived the idea of a torus consisting of six test coils, externally compatible but exploring various designs of conductor and structure. Similar interests in other countries led in 1977-78 to the establishment of the LCT.
The goal of the LCT was stated in these words: "to obtain experimental data, to demonstrate reliable operation of large superconducting coils, and to prove the design principles and fabrication techniques proposed for the magnets in a tokamak experimental power reactor". This goal was to be achieved by largely independent design, development and construction of six test coils, followed by extensive, collaborative testing in a compact toroidal array.

The four LCT parties were EURATOM, Japan Atomic Energy Research Institute (JAERI), the Swiss Département Fédéral de l'Intérieur, and the U.S. Department of Energy (DOE). Under the terms of an International Energy Agency (IEA) agreement, DOE served as the LCT Operating Agent, building and operating the required test facility at its Oak Ridge National Laboratory (ORNL). DOE also provided three different test coils while the other three participants provided one coil each. Government laboratories and industrial partners or contractors were involved as shown in Fig. 1. Fig. 2 shows the arrangement of coils in the test stand.
Fig. 3, showing one the coils being installed at Oak Ridge, gives an impression of their dimensions. Each coil is about $5 \mathrm{~m}$ high and weighs about $40,000 \mathrm{~kg}$. The size and shape were specified to leave only reasonable extrapolations of LCT fabrication methods to those for full-size tokamak reactor toroidal field coils.

Coil specifications ensured compatibility with the test array and demanded reactor-relevant performance but allowed each design team freedom to make some important design choices. The facility was designed for cooling coils with either a $4.2 \mathrm{~K}$, atmospheric pressure bath of helium (pool-boiling) or a flow of high pressure helium (forced-flow). The peak field requirement was set at $8.0 \mathrm{~T}$ at the winding, which was considered to be near the practical limit for NbTi conductors at $4.2 \mathrm{~K}$ and attractive current density. Coils had to be designed with enough tolerance towards internal heating to continue operation despite foreseeable perturbations. The designs also contemplated operation with a distributed load resembling nuclear heating in a tokamak coil and, in addition, in the

Fig. 1 - Responsibilities in the International Energy Agency's Large Coil Task.

INTERNATIONAL ENERGY AGENCY

LARGE COIL TASK

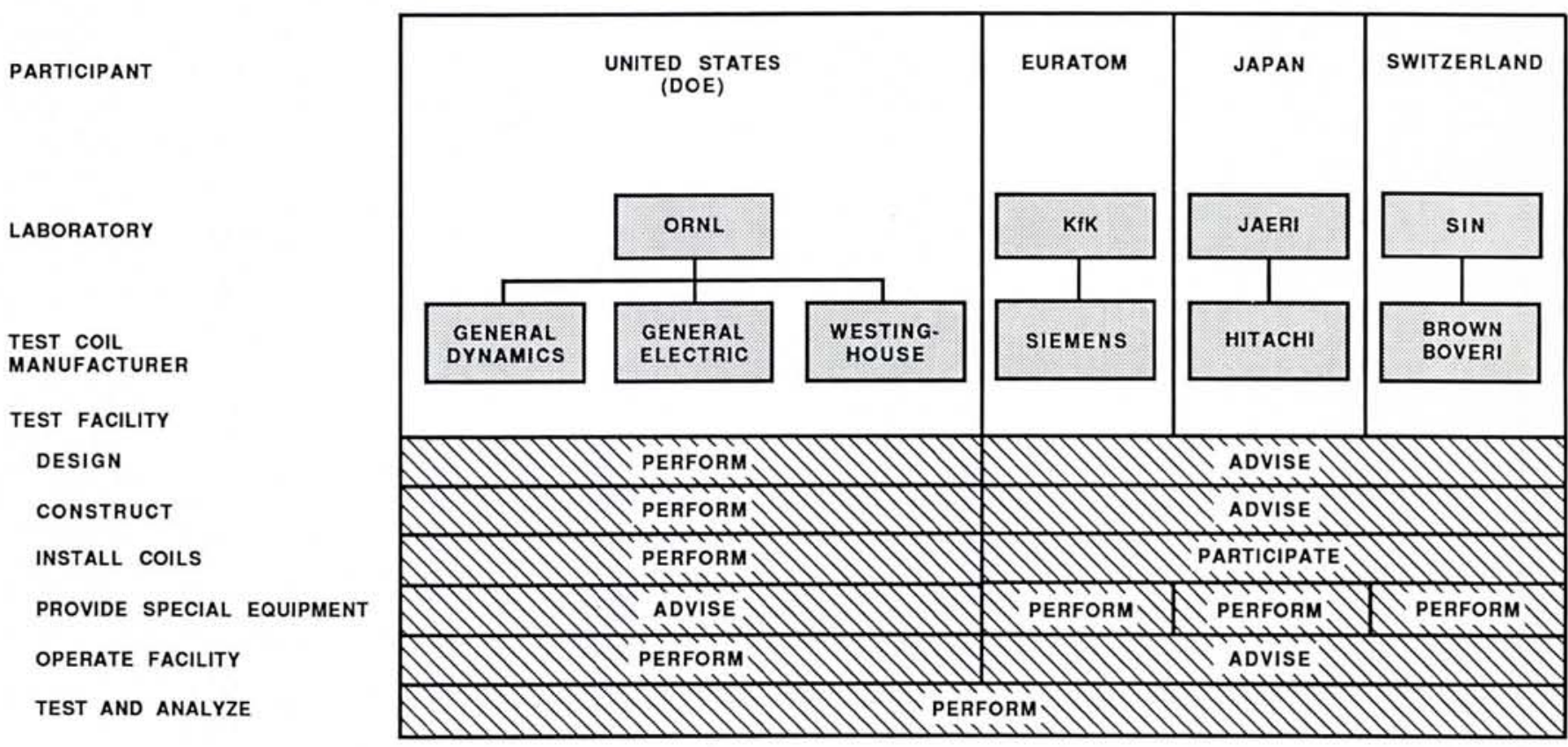



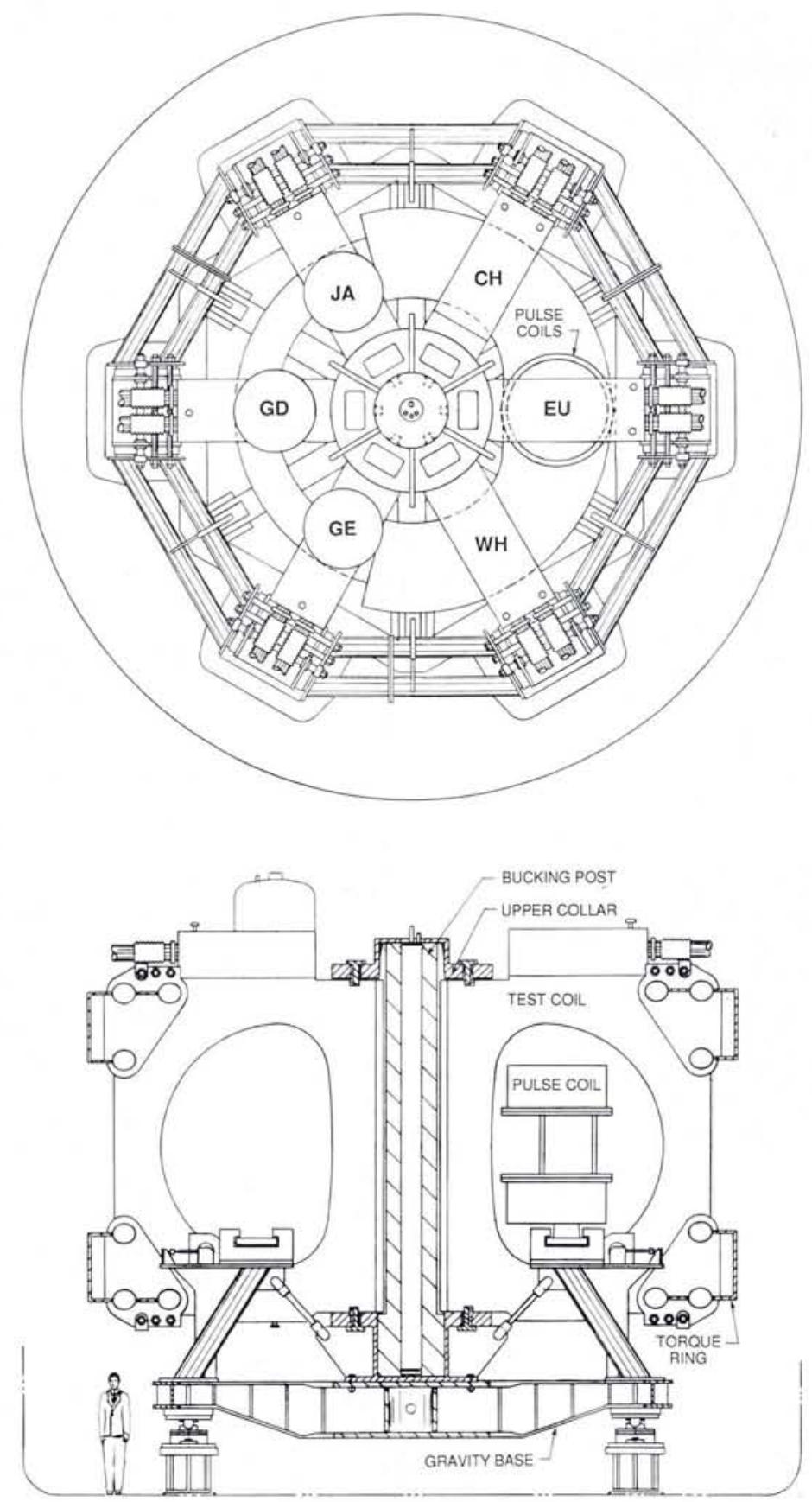

Fig. 2 - Arrangement of coils in the LCT test stand.

presence of an externally imposed pulsed field.

Three of the teams chose pool-boiling; three, forced-flow. Five coils used $\mathrm{NbTi}$ conductors; one, $\mathrm{Nb}_{3} \mathrm{Sn}$. The wide variety of configurations for the conductors is illustrated in Fig. 4. There were also significant differences in the design of coil structure and insulation and in fabrication methods. Considerations of stability, coil protection and manufacturing capabilities led design teams to choose conductor current ratings in the range 10.2 to $10.5 \mathrm{kA}$ for the bathcooled coils and 11.4, 13.0 and $17.8 \mathrm{kA}$ for the forced-flow coils (EU, $\mathrm{CH}$ and WH respectively). The total current in each coil, required to reach the $8 \mathrm{~T}$ design
$\mathrm{Nb}_{3} \mathrm{Sn}$ coil) was completed. The $420 \mathrm{t}$ array of six coils and supporting structure was cooled to operating temperature in January-February 1986. For almost two years the array remained under vacuum in an $11 \mathrm{~m}$ diameter tank, while extensive testing was performed as planned.

\section{Test Programme}

In the first series of tests, one coil at a time was energized to its rated current. No evidence of training was observed and no impediment to operation was encountered. These "single-coil" tests included various experiments to verify the stability of the coils. In some of these, coils recovered the fully superconductive state after a full turn of conductor was driven normal by heaters embedded in the winding.

Next were design-point tests in which all six coils were operated simultaneously. In each test, one coil was designated the "test coil" and was connected for the maximum degree of signal processing and data acquisition. The other five "background" coils were brought up to about 0.8 of rated current while the test coil was being charged to rated current, which produced a field of $8.0 \mathrm{~T}$ at the test coil's winding. Again "nuclear" heating, stability and dump tests were performed.

Three of the coils (Euratom, Swiss and Japanese) were tested at high current while a series of vertical field pulses were applied. Heating effects were satisfactorily low, in reasonably good agreement with design calculations. The actuating mechanisms for moving and locking the pulsed coils failed before pulsed-field tests could be performed on the other coils.

As planned, the limits of operability of each coil were determined by "extended condition" tests. In one set of tests, each coil was energized singly to determine the highest attainable current in selffield. The maximum currents in these tests ranged from 120 to $140 \%$ of the $8 \mathrm{~T}$ rating. The $\mathrm{Nb}_{3} \mathrm{Sn}$ coil was operated at rated current with its coolant inlet temperature at $8 \mathrm{~K}$, more than twice its usual $3.8 \mathrm{~K}$. In the concluding test, currents in all six coils were raised simultaneously until a limiting condition was reached. This proved to be an overloading of the refrigerator by the resistive heating in parts of the $\mathrm{Nb}_{3} \mathrm{Sn}$ conductor. The coolant temperature began to rise and soon the $\mathrm{Nb}_{3} \mathrm{Sn}$ quenched. At this point the peak field in each coil was at 9.0 T or slightly higher. In this test the stored energy reached $944 \mathrm{MJ}$, which was dissipated harmlessly in external dump resistors.
The coil design work started in 1977 .

78 and in 1985 the last of the coils (the 


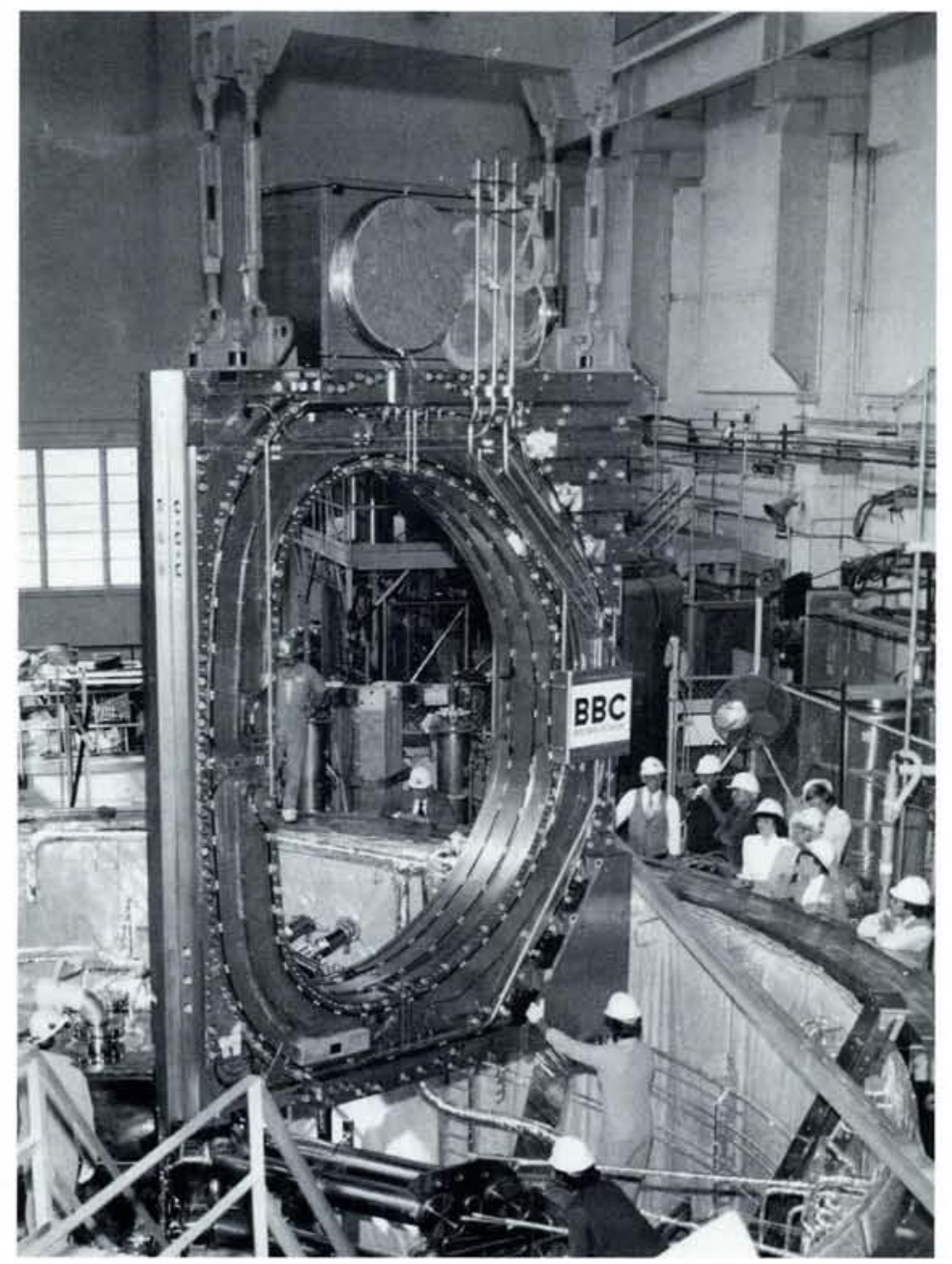

Fig. 3 - Swiss coil being installed in the International Fusion Superconducting Magnet Test Facility at Oak Ridge.

Coil energy dumps were executed numerous times during the test programme. Some were done specifically for the purpose of obtaining data on coil behaviour during a dump. Others were initiated automatically whenever a "normal" zone appeared during an extended condition test. No damage was sustained.

Responses of winding and structure to abnormally high out-of-plane loads were observed in tests in which five coils were run up to high current while one remained de-energized. All coils performed satisfactorily, without damage to the windings or excessive structural strains.

Tests beyond design points included so-called "safety tests", designed to ascertain the stringency of requirements for control actions to prevent damage in case of unusual occurrences. Some tests involved production of propagating normal zones while the automatic dump was delayed to allow "hot spots" to develop. Others determined the behaviour of a forced-flow coil when flow was stopped while the coil was operating at rated current. Results showed remar- used to specify control actions were quite conservative.

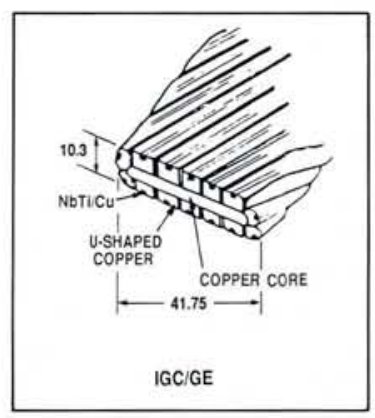

The sustained operation of the facility provided useful information on performance and availability of vital supporting systems: helium refrigeration, liquid nitrogen and vacuum systems, cryogenic instrumentation, etc. Performance was generally quite satisfactory, as all systems provided their intended functions except for the equipment for remotely moving the pulse coils. The availability of the test facility was defined as the fraction of time spent on planned programme activities. Over the 22 months from October 1985 to September 1987, which spanned start-up and the entire 6 -coil test programme, the availability factor was 0.57. By far the largest single cause of downtime, i.e., unavailability, was air leakage into the helium. This occurred in sections operated at subatmospheric pressure in order to reduce the temperature of the helium supply to the forced-flow coils to $3.8 \mathrm{~K}$.

\section{Conclusions}

Results were quite gratifying, as each of the coils demonstrated excellent performance, operated quite reliably, and produced definitive data on behaviour under a wide variety of conditions. Advantages and limitations of the various design features were clearly brought out, but no one coil was identified as superior or inferior overall.

In their final joint analysis, the four LCT participants agreed on statements of findings or conclusions from the programme, including the following.

- Each coil achieved and significantly exceeded design-point performance.
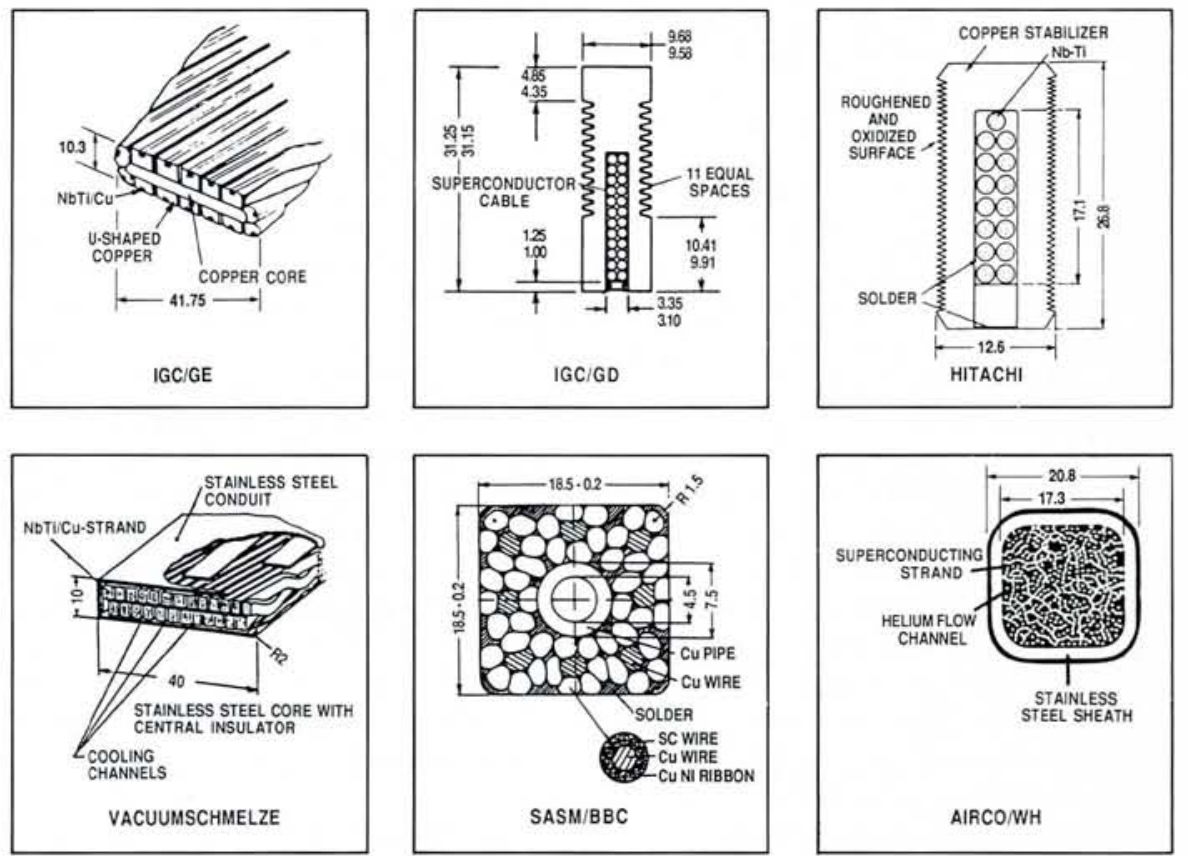

Fig. 4 - Configurations of conductors in LCT coils.
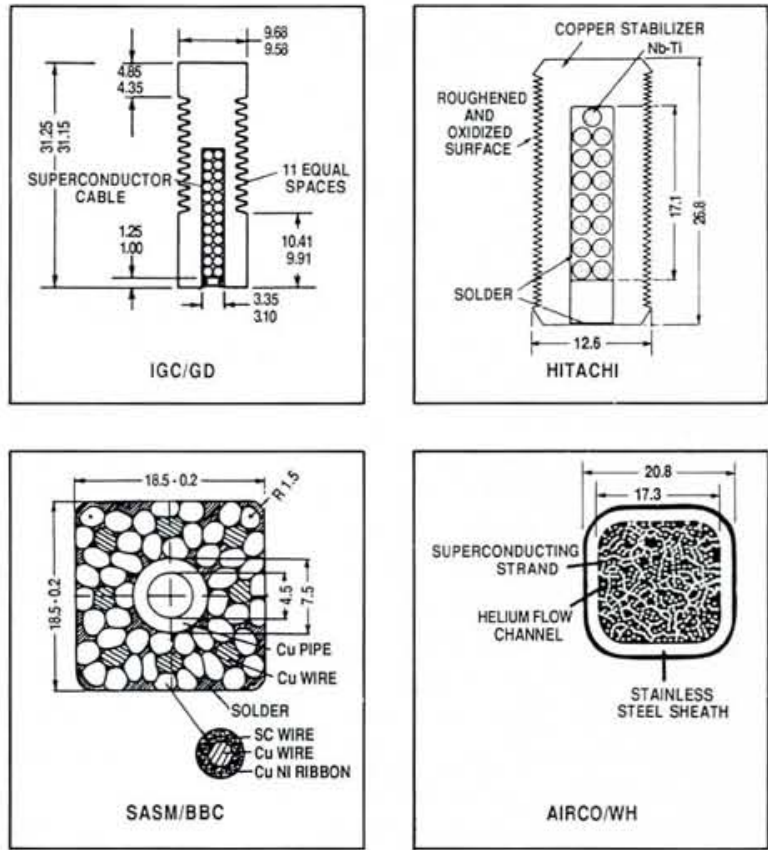

DIMENSIONS IN mm 
- The ample diagnostic instrumentation provided data that enabled analyses of electrical, mechanical, and thermal behaviour in more detail than in any previous test of superconducting magnets.

- The mature state of development of $\mathrm{NbTi}$ superconductors was demonstrated again.

- The current-carrying ability of the $\mathrm{Nb}_{3} \mathrm{Sn}$ conductor fell short of expectations because of imperfections scattered along the $4 \mathrm{~km}$ length in that coil, which indicated that more $R \& D$ on $\mathrm{Nb}_{3} \mathrm{Sn}$ conductor is needed.
- The practicality of both pool-boiling and forced-flow cooling for toroidal field coils of this size was demonstrated.

- Satisfactory stability of much larger tokamak magnets should be achievable through the use of the design procedures tested in the LCT.

- Evaluation of LCT results, in conjunction with other magnet $R \& D$ results, suggests that forced-flow cooling is preferable for coils much taller than those in LCT. Further R\&D is needed, however, on the stability of coils with longer cooling channels.
- Effective collaboration in LCT, involving integration of large-scale, advanced-technology components that were co-operatively designed and produced in several countries, presages success in larger ventures, such as the International Thermonuclear Experimental Reactor (ITER).

\section{REFERENCE}

[1] Haubenreich P.N. et al., The IEA Large Coil Task-Development of superconducting toroidal field coils for fusion power, Fusion Engrg. and Design 7 (1988) pp 1-230.

\section{Inertial Confinement Fusion}

\section{A report from BEAMS' 88 : \\ 7th International Conference on High-Power Particle Beams}

The BEAMS'88 conference, held on 4-8 July, 1988, at the Karlsruhe congress centre, FRG, was organized by the Karlsruhe Nuclear Research Center (Chairmen: Prof. A. Citron and Prof. G. Kessler) - with the sponsorship of five scientific institutions (EPS, Univ. of Karlsruhe, DPG, IEEE German Section, $\mathrm{VdE})$. BEAMS' 88 was the 7 th of a series of international conferences held every two years. About 300 participants from leading laboratories from all over the world presented 215 original research papers and 12 review papers in 14 oral sessions (single-sessions!) and 33 poster sessions.

As in earlier BEAMS conferences, a central topic was the scientific and technological progress in approaches to inertial confinement fusion (ICF) which are based on pulsed particle beams (micro pellet fusion, especially with light-ion beams) and pulsed power discharges (plasma foci, liner implosions, z-pinches).

According to present estimates, a driver for break-even experiments with particle or laser beams must deposit an energy of 5-10 MJ on a target of less than $1 \mathrm{~cm}$ in diameter in a period of less than $30 \mathrm{~ns}$. For the ICF approach using particle beams, an essential step on the way towards reaching this objective was the recent installation of the PBFA II fusion device at Sandia. The goal is to generate a lithium ion beam of approximately 100 TW in power and more than $1 \mathrm{MJ}$ in energy and with it to verify ICF ignition physics. During the test experiments carried out on the accelerator with reduced voltage, and reported at the conference, ion pulses of up to 0.5 MJ beam energy were generated. The focussing of the ion beam was still im- perfect, so that target experiments will have to wait until the ion diodes used for beam generation have been further improved. Various ion diode concepts have been tried in the past. Some proved successful at power levels $\lesssim 1 \mathrm{TW}$ and the problems arising at higher power levels still, do not seem unsurmountable. Recent developments in diagnostic methods broaden the scientific and technological knowledge needed for the construction of high-focussing light-ion diodes with multiterawatt beam power.

In the USSR, the ICF approach with liner implosions where a high current discharge is produced in the thin solid or gaseous walls of a hollow cylinder, which then implodes, has obtained high priority in particular at the Kurchatov Institute in Moscow. In this approach, the soft $X$-ray hohlraum radiation generated in an imploding liner discharge illuminates a fusion pellet which implodes in turn so that fusion conditions are reached. The object of these experiments which are carried out at the large ANGARA- $V-1$ pulsed power generator, is to obtain stable implosions.

Impressive results were also reported from the Japanese laser programme at Osaka where considerable improvements in the quality of pellet implosions by lasers had been achieved. This was a very important contribution since it underlines the need for giving the highest priority to the development of large scale drivers for break-even experiments, in particular, ion diodes.

Breakthroughs in implosion experiments of pellets by X-rays were claimed by the American side in as yet unpublished experiments, summarized by the representative of the Department of Energy, S. Kahalas, in his statement:

"ICF is not a question of the "if", it is only of the "when"".

There were various other fields to which the scientific topics of this BEAMS conference had been dedicated: high-power charged particle beams (including cluster beams) for science and industry (including magnetic confinement fusion), high-voltage pulsedpower generators, interaction of particle beams with matter and diagnostics methods, free electron lasers, generation of high-power $\mathrm{X}$ - and $\gamma$-radiation, and generation of high-power microwave radiation. (The latter topic had a historical relation to the BEAMS' 88 conference date and venue: in 1888, 100 years before BEAMS' 88 , Heinrich Hertz had discovered the electromagnetic waves at Karlsruhe, - in the form of low-power coherent microwaves.) It is not the purpose of this article to review the large amount of progress reported in these fields. The interested reader is invited to request a free copy of the BEAMS'88 Book of Abstracts from: Prof. G. Kessler, KfK, P.O. Box 3640, D-7500 Karlsruhe 1, W. Germany. The 2-volume BEAMS'88 Proceedings with complete texts of all conference papers can be obtained from the Literaturabteilung at the same address; price: DM 120.-.

BEAMS' 88 has emphasized the liveliness of these fields. Great hopes were expressed for further scientific breakthroughs before the community will meet again at BEAMS'90, which will take place at Novosibirsk, USSR, in the summer.

H.U. Karow Organizing Secretary of BEAMS' 88
W. Schmidt Scientific Secretary of BEAMS' 88 